\title{
Design and Analysis of Multilayered Waveguide Structure with Metal-Dielectric Gratings for Sensing with Reflection Narrowband Notch Filter
}

\author{
Guiju ZHANG ${ }^{1,2,3}$, Bing CAO ${ }^{1,2 *}$, Ke ZHANG ${ }^{1,2}$, Chinhua WANG $^{1,2}$, Qian SUN $^{4}$ \\ ${ }^{1}$ College of Physics, Optoelectronics and Energy, Soochow University, Suzhou 215006, China \\ ${ }^{2}$ Key Lab of Advanced Optical Manufacturing Technologies of Jiangsu Province\& Key Lab of Modern Optical \\ Technologies of Education Ministry of China, Soochow University, Suzhou 215006, China \\ ${ }^{3}$ CREOL, The College of Optics \& Photonics, University of Central Florida, Orlando, FL, 32816, USA \\ ${ }^{4}$ Suzhou Institute of Nano-tech and Nano-bionics, Chinese Academy of Sciences, Suzhou, 215125, China
}

cross $^{\text {ref }}$ http://dx.doi.org/10.5755/j01.ms.21.4.9625

Received 27 January 2015; accepted 11 September 2015

\begin{abstract}
Developments in micro and nanofabrication technologies have led a variety of grating waveguide structures (GWS) being proposed and implemented in optics and laser application systems. A new design of multilayered nanostructure double-grating is described for reflection notch filter. Thin metal film and dielectric film are used and designed with one-dimensional composite gratings. The results calculated by rigorous coupled-wave analysis (RCWA) present that the thin metal film between substrate and grating can produce significant attenuated reflections and efficiency in a broad reflected spectral range. The behavior of such a reflection filter is evaluated for refractive index sensing, which can be applied inside the integrated waveguide structure while succeeding cycles in measurement. The filter peaks are designed and obtained in a visible range with full width half maximum (FWHM) of several nanometers to less than one nanometer. The multilayered structure shows a sensitivity of refractive index of $220 \mathrm{~nm} / \mathrm{RIU}$ as changing the surroundings. The reflection spectra are studied under different periods, depths and duty cycles. The passive structure and its characteristics can achieve practical applications in various fields, such as optical sensing, color filtering, Raman spectroscopy and laser technology.

Key words: notch filter, multilayered nanostructure, double-grating, reflected attenuation, refractive index sensing.
\end{abstract}

\section{INTRODUCTION}

Resonant narrowband filters using periodic grating nanostructures have attracted much attention owing to their high peak reflection and transmission, low sideband and controllable wavelength peak and width [1-3]. Guidedmode resonance filters based on grating waveguide structures (GWS) with different constitution and materials are candidates for extremely narrow line filters or color filters [4-5]. In conventional dielectric mirror design, high reflectors can be designed only on multilayered coatings, which are made from optically isotropic and homogeneous materials. The so-called quarter-wave stack consists of alternating high- and low-refractive index layers of quarter-wave optical thickness at given reference wavelength. In contrast, GWS is with specially designed uniform and grating layers to achieve such tailored optical properties, which is accomplished parallel to the film surface. However, the multilayered structures with gratings do not need to be particularly thick under the necessary quarter-wave condition. It is suitable for such a multilayered structure being an alternative and comfortable notch filter.

In any case, the rejection bandwidth is greatly influenced by the refractive indices of materials, the geometry parameters of multilayered structures and the realization of a given specific filter depends on the availability of suitable and optimal designed structures.

\footnotetext{
* Corresponding author. Tel.: +86-512-65113371;

fax: +0512-65112232. E-mail address: bcao2006@163.com (B. Cao)
}

Such filters can be designed with alternative resonant wavelength peak and bandwidth, which have been explored for switch and laser cavity reflector applications in communication and laser systems. Optical properties of the filters, such as the resonance wavelength and linewidth, peak reflection or attenuation, are heavily dependent on the nanostructures parameters (period, filling factor and depth) and materials. The dimensional parameters can be designed and optimized to achieve different particular resonant filters, which are good choices for novel sensors, spectroscopic compression and laser technology [6-8].

The purpose of this paper is to demonstrate the feasibility of multilayered waveguide structures with significantly narrow reflections by integrating doublegrating layer. Characteristics of both TM reflection spectra and resonance peak of the multilayered notch filtering devices with different structure parameters are investigated under different surroundings. The structure shows a high sensitivity to the refractive index of media. Notch filtering properties of the multilayered nanostructure with different thin film layers and subwavelength grating layers are calculated and optimized within the framework of Rigorous Coupled-Wave Analysis (RCWA) [9-10].

\section{MULTILAYERED WAVEGUIDE STRUCTURE WITH NARROWBAND REFLECTED ABSORPTION}

Grating waveguide structures with integrated subwavelength gratings and thin layer films present transmission or reflection narrowband filtering properties. 

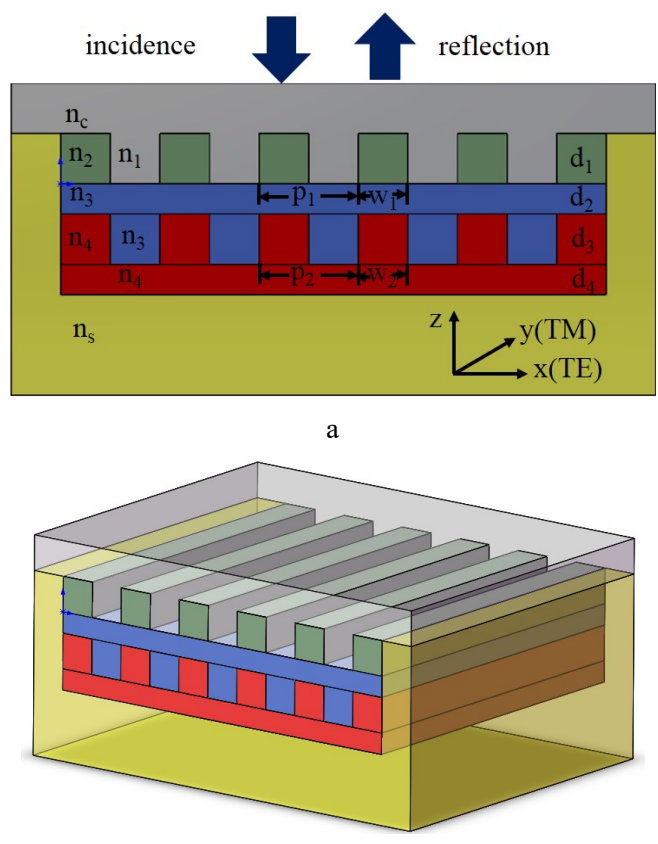

b

Fig. 1. Principle structure of a multilayered grating waveguide: $a-$ section view of the structure; $b$-perspective view of the structure

A multilayered absorption reflector is built up by a thin metal film with one-dimensional gratings and layers on top, which consists of two different dielectrics and one metal; as seen in Fig. 1. The bottom substrate is silica with $n_{\mathrm{s}} \sim 1.46$ and the cover is with refractive index $n_{\mathrm{c}}$. There are four layers between substrate and cover: a metal thin layer with refractive index $n_{4}$ and depth $d_{4}$; a bottom metal / dielectric compound grating layer with different refractive index $n_{4}$ and $n_{3}$, depth $d_{3}$, period $p_{2}$ and metal stripe width $w_{2}$; a dielectric layer with $n_{3}$ and depth $d_{2}$; a surface composite dielectric grating layer with different refractive indices $n_{2}$ and $n_{1}$, depth $d_{1}$, period $p_{1}$ and the stripe $n_{2}$ and width $w_{1}, n_{1}$ can always be the same as $n_{\mathrm{c}}$. The metal film and stripe can be set as aluminum, silver or copper and dielectric film and stripe can be optical materials as $\mathrm{SiO}_{2} \backslash \mathrm{MgF}_{2} \backslash \mathrm{ZnS} \backslash \mathrm{Al}_{2} \mathrm{O}_{3}$. Duty cycle (DC) is defined as the filling factor of metal or one of the dielectrics the grating. In the simulation, the surface and the bottom grating period is separately set as $p_{1}$ and $p_{2}$ and the rectangular stripe width is $w_{1}$ and $w_{2}$, so $\mathrm{DC} 1=w_{1} / p_{1}$ and DC2 $=w_{2} / p_{2}$. The value of duty cycle is less than 1.0. Fig. $1 \mathrm{a}$ shows the sectional view of the multilayered nanostructure, and Fig. $1 \mathrm{~b}$ shows the perspective structure. The incident angle is defined in a way from the $\mathrm{z}$ axis to $\mathrm{x}$ axis, that is plane (ZOY) is the oblique incidence plane. The incident plane wave is specified with wavelength and propagation direction, $\mathrm{z}$-axis is the light reflected direction of incidence, $\mathrm{x}$-axis and $\mathrm{y}$-axis is defined as p-polarized $(T M)$ and s-polarized (TE) direction, respectively. The vector of electric field is parallel to the plane of the grating vector ( $\mathrm{x}$ axis) for TM polarized state and perpendicular to the plane of the grating vector for TE polarized state. With an appropriate metal/ dielectric grating design, such a multilayered waveguide structure with gratings reflects TM polarization with resonant attenuations, and exhibits excellent reflection notch filtering behavior. TM polarization is analyzed and calculated for the reflection output as follows.

In conventional multilayered planar waveguide, the resonance will happen according to the resonance wavelength conditions, which the layer thickness is a multiple of half-wavelength [2]. In the multilayered waveguide structure with gratings, strong interaction resonance happens as a diffracted order is phase matched to a leaky mode, that is, the layer doesn't need to be in a particularly thickness [10]. The real part guided mode propagation constant is near the diffracted order propagation constant:

$\beta_{\text {real,mode }} \cong \frac{2 \pi}{\lambda_{0}} n_{\text {inc }} \sin \theta_{\text {inc }}-\frac{2 \pi}{p}$,

where, $n_{\text {inc }}$ is the refractive index of incidence material, $\Theta_{\mathrm{inc}}$ is the incident angle; $\lambda_{0}$ is the resonance wavelength, $p$ is the grating period. The resonance results in narrowband reflection peak at the phase matching wavelength. The peak wavelength and the reflection width are mainly determined by the grating period, duty cycle, thickness, angle of incidence and refractive index. In particularly, a very thin metal film is used between the substrate and the bottom metal / dielectric grating for resonance reflections. In such a GWS, the propagation constant is complex, thus the mode is leaky with certain wavelength. The resonance structure couples the light into the leaky guided modes, which shows visible in the reflection spectra as attenuated peaks [13]. The design goal for the device is to achieve as resonance attenuation wavelength as possible. In the present work, the behaviors of the proposed structures are characterized by using Gsolver, which is based on a full vector implementation of rigorous coupled wave theory [11-12].

The multilayered waveguide structure thus possesses a notch reflection filter spectral characteristic in a broad spectral range. Fig. 2 a shows different reflection resonances in wavelength under multilayered structures with different parameters.

The resonant attenuation peak is separately $541.65 \mathrm{~nm}$, $547.05 \mathrm{~nm}$ and $551.65 \mathrm{~nm}$ with $0.6 \mathrm{~nm}, 0.45 \mathrm{~nm}$ and $0.35 \mathrm{~nm}$ in FWHM, as seen in insert figure. Structures G1 and G2 are with the parameters as: $n_{4}$ is aluminum and $d_{4}=20 \mathrm{~nm} ; n_{3}=1.5$ and $d_{3}=240 \mathrm{~nm}$ with $p_{2}=340 \mathrm{~nm}$, $\mathrm{DC} 2=0.5 ; d_{2}=240 \mathrm{~nm} ; n_{2}=2.3(\mathrm{ZnS}), d_{1}=240 \mathrm{~nm}$ and $\mathrm{DC} 1=0.4$ with $p_{1}=p_{2} ; n_{\mathrm{c}}=1.35$. The only difference between these two double-grating multilayered structures is: $n_{1}=n_{c}$ for $\mathrm{G} 1$ and $n_{1}=1.38(\mathrm{MgF} 2)$ for G2. Structure G3 is designed as: $n_{4}$ is aluminum, $n_{3}$ is silica and $n_{1}=n_{\mathrm{c}}=1.0 ; d_{4}=10 \mathrm{~nm}, d_{3}=300 \mathrm{~nm}$ and $d_{2}=160 \mathrm{~nm}$; $p_{2}=300 \mathrm{~nm}$ and DC2 $=0.5$, the first layer under cover is a uniform film with $n_{2}=2.3, d_{1}=140 \mathrm{~nm}$. The input light is under normal incidence. Absorption wavelength peak shifts sensitively to the surroundings, which can be evaluated by the behavior of a refractive index sensor. Fig. $2 \mathrm{~b}$ shows absorption peak shift results as the surrounding $n_{\mathrm{c}}$ is changed from 1.33 to 1.35 for the doublegrating structure G1 and G2. The peaks present red shifts from $538.1 \mathrm{~nm}$ to $541.65 \mathrm{~nm}$ with a shift of $3.55 \mathrm{~nm}$ for G1, from $546.45 \mathrm{~nm}$ to $547.05 \mathrm{~nm}$ with a shift of only $0.6 \mathrm{~nm}$ for G2. More detailed resonance and wavelength 
properties are acquired and discussed in the following section.
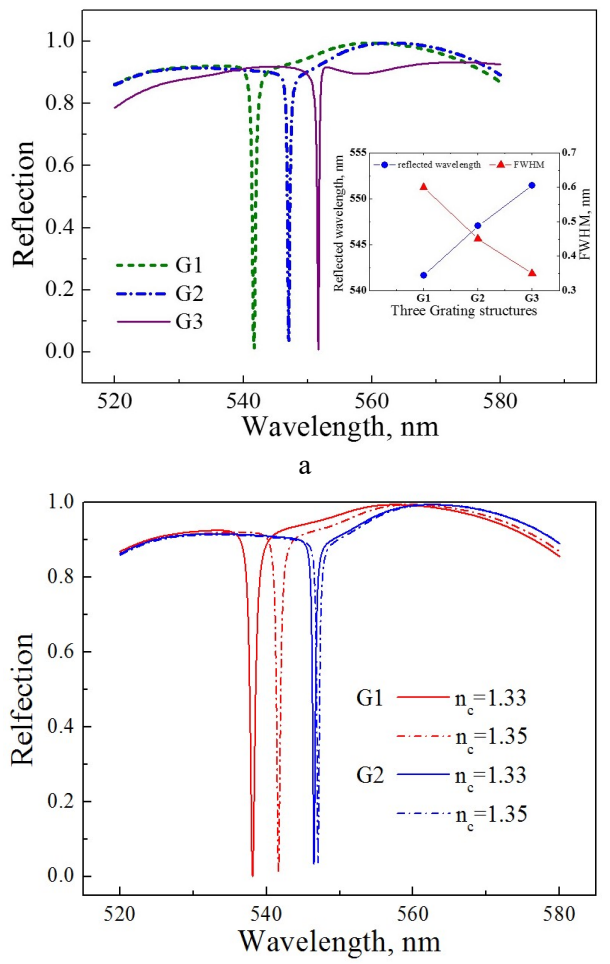

b

Fig. 2. Reflection spectra in different wavelength: a-different attenuation wavelength and FWHM reflections; $b-$ reflection peak shifts in different surroundings

\section{SIMULATION RESULTS AND DISCUSSION}

The performance of the multilayered waveguide structures with gratings is characterized by their resonance wavelength (absorption peak), bandwidth and reflection. In this section, we carefully design these characteristics to meet the required performance, and discuss the absorption spectra properties of wavelength peak and width based on such a multilayered GWS shown in Fig. 1. The attenuated resonance peak of wavelength is saliently determined by the grating period. Fig. 3 a shows different resonant attenuation reflection peaks in a wide wavelength range of $400 \mathrm{~nm}$ under different grating periods. The multilayered grating structure reveals a red shift of the peak as the grating period increases. The absorption peak changes from $457.85 \mathrm{~nm}$ to $758.1 \mathrm{~nm}$ as period is from $240 \mathrm{~nm}$ to $430 \mathrm{~nm}$ under normal incidence. The longer period the longer absorption wavelength peak. The grating period is an important factor that influences the absorption peak. Here, $n_{1}=n_{\mathrm{c}}=1.33$. The double grating layers with the same period and the duty cycle $\mathrm{DC} 1=\mathrm{DC} 2=0.5$. The absorption peak can be designed and optimized at different wavelengths and widths; be coincidence with the light source and detector. Fig. $3 \mathrm{~b}$ shows peak shift changes as $n_{\mathrm{c}}$ is changed from 1.33 to 1.35 under different periods. The longer period of the grating, the more sensitive red peak shift to the surroundings.

The optical properties and the changes of resonant attenuation peak are calculated under oblique incidence in Fig. 4. Two different multilayered double-grating structures G4 and G5 are designed both on substrate silica. We separately use $\mathrm{ZnS}$ and the surrounding material $n_{\mathrm{c}}\left(n_{1}=n_{\mathrm{c}}\right)$ for the surface grating of $\mathrm{G} 4, \mathrm{ZnS}$ and use $\operatorname{MgF} 2\left(n_{1}=1.38\right)$ for the surface grating of G5.

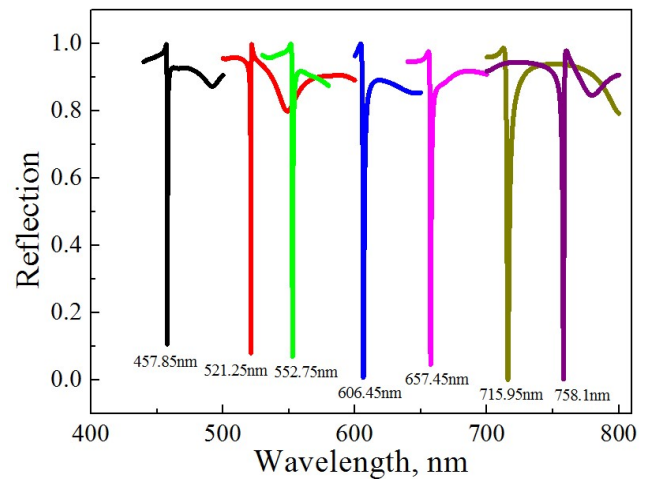

a

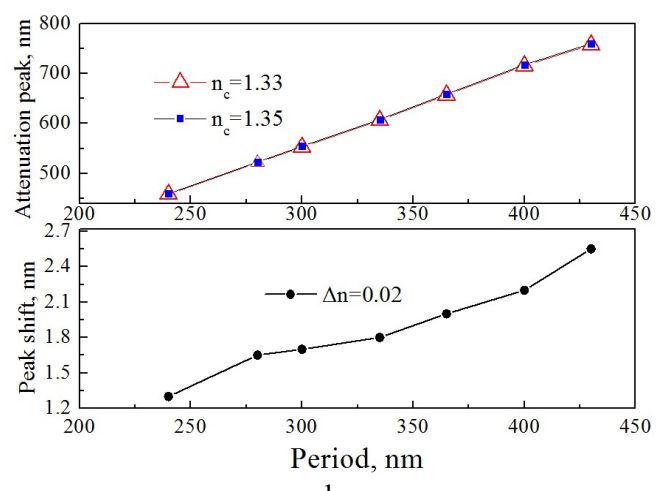

b

Fig. 3. Reflection spectra under different grating period: a-different attenuation reflection wavelength; $\mathrm{b}$-reflection peak shifts in different surroundings

The structural parameters for both are set as: Aluminum film on the substrate with thickness $d_{4}=20 \mathrm{~nm}$, the bottom grating $\mathrm{Al} / \mathrm{SiO}_{2}$ with $p_{2}=300 \mathrm{~nm}, \mathrm{DC} 2=0.5$ and $d_{3}=240 \mathrm{~nm}$, silica film with $d_{2}=180 \mathrm{~nm}$, the surface grating layer with $p_{1}=300 \mathrm{~nm}$ and $\mathrm{DC} 1=0.5$.

The attenuation peaks of the notch filter shift towards the longer wavelength as the oblique angle increases, as seen in Fig. 4 a. Peak shifts from $553.4 \mathrm{~nm}$ to $594.5 \mathrm{~nm}$ with the angle changing from $15^{\circ}$ to $25^{\circ}$ for $\mathrm{G} 4$, from $523.6 \mathrm{~nm}$ to $585.3 \mathrm{~nm}$ with $10^{\circ}$ to $25^{\circ}$ for G5. Where, the surrounding is with refractive index $n_{\mathrm{c}}=1.33$. When $n_{\mathrm{c}}$ is changed to 1.35 with oblique incidence of $25^{\circ}$, the peak shifts in red respectively to $598.9 \mathrm{~nm}$ and $588.9 \mathrm{~nm}$ for G4 and G5. These two filters show high sensitivities of refractive index with $\sim 220 \mathrm{~nm} / \mathrm{RIU}$ and $180 \mathrm{~nm} / \mathrm{RIU}$, as seen in Fig. 4 b. The larger incident angle makes the larger peak deviation within the same refractive index changes.

More resonant absorption properties are studied under various structure parameters, e.g. grating duty cycle and grating is changed only. Here, the oblique incidence angle is set as $20^{\circ}$. The attenuation peak shows a red shift as the filling factor of high index material increases, as seen in Fig. $5 \mathrm{a}$. The resonance peak shifts from $554.6 \mathrm{~nm}$ to $581.2 \mathrm{~nm}$ and from $558.7 \mathrm{~nm}$ to $584.7 \mathrm{~nm}$ as the duty cycle changes from 0.4 to 0.6 . Where $n_{\mathrm{c}}$ equals 1.33 and 1.35 separately. The FWHM of such a notch filter reduces quickly from $5.3 \mathrm{~nm}$ to $1.0 \mathrm{~nm}$ as duty cycle increases. 


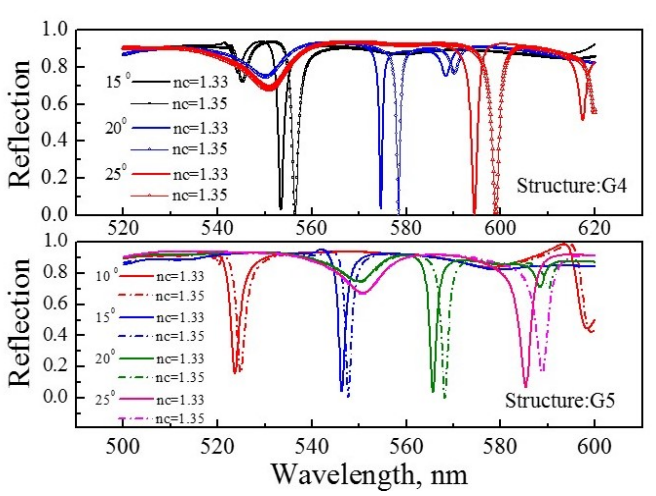

a

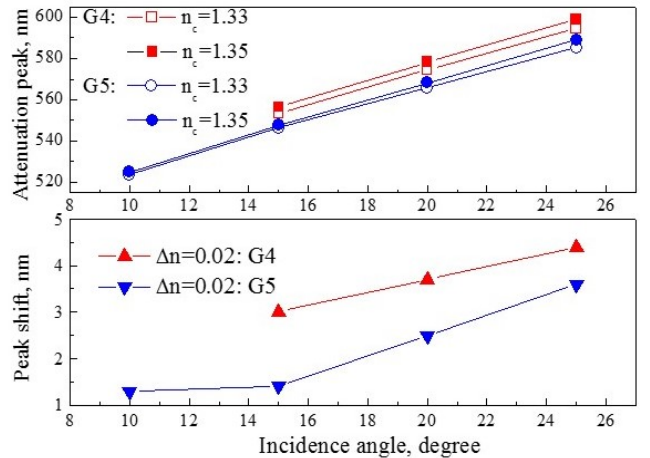

Fig. 4. Reflection spectra under different incidence angle: a- different attenuation reflection wavelength; $\mathrm{b}$-reflection peak shifts in different surroundings
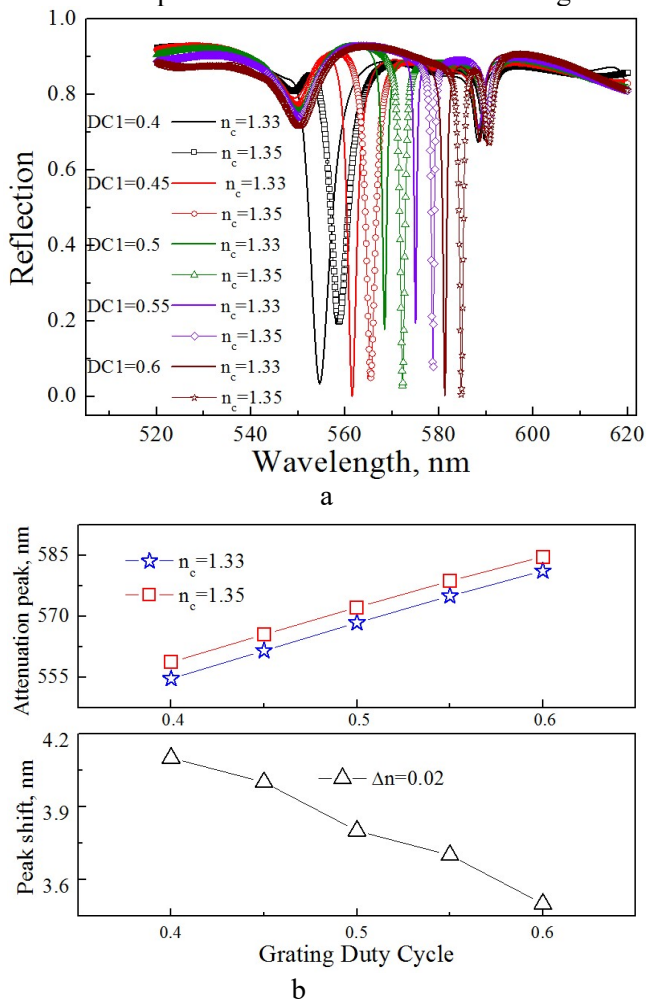

Fig. 5. Reflection spectra under different duty cycle: a-different attenuation reflection wavelength; $\mathrm{b}$-reflection peak shifts in different surroundings

Fig. 5 gives the absorption spectra results for structure G4 as DC1 of the surface. The structure shows less sensitivity to the surroundings as the surface grating duty cycle increases, as seen in Fig. 5 b.

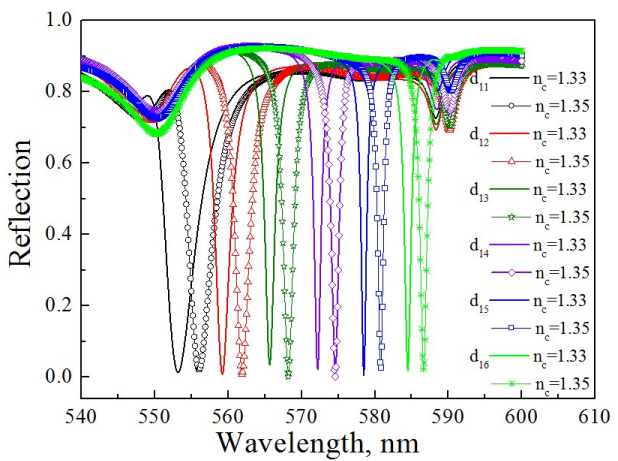

a

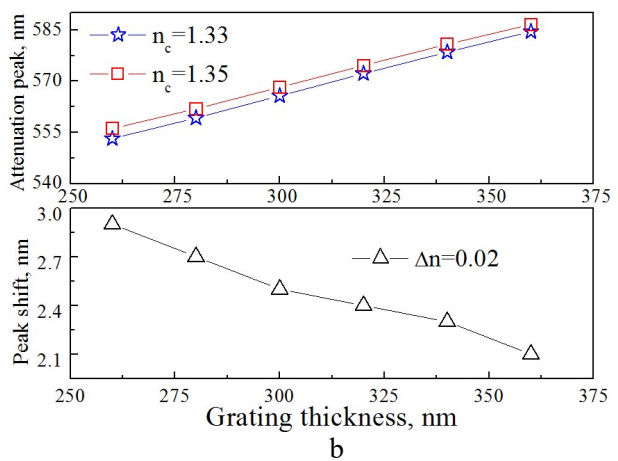

Fig. 6. Reflection spectra under different grating depth: a- different attenuation reflection in wavelength; $\mathrm{b}$-reflection peak shifts in different surroundings

The peak deviation decreases from $4.1 \mathrm{~nm}$ to $3.5 \mathrm{~nm}$ as duty cycle increases from 0.4 to 0.6 within a refractive index deviation of 0.02 , thus, the refractive index sensitivity decreases from $205 \mathrm{~nm} / \mathrm{RIU}$ to $175 \mathrm{~nm} / \mathrm{RIU}$.

Different grating depths also affect the resonant absorption spectra, peak shift and deviation. Fig. 6 shows the results for structure G5 under different grating depth $d_{1}$. The reflection peak presents a red shift as the grating depth increases from $d_{11}=260 \mathrm{~nm}$ to $d_{16}=360 \mathrm{~nm}$, seen in Fig. 6 a. The peak wavelength changes from $553.2 \mathrm{~nm}$ to $584.5 \mathrm{~nm}$ in the surrounding $n_{\mathrm{c}}=1.33$, and from $556.1 \mathrm{~nm}$ to $586.6 \mathrm{~nm}$ in surrounding $n_{\mathrm{c}}=1.35$. The FWHM reduces quickly from $5.3 \mathrm{~nm}$ to $1.2 \mathrm{~nm}$ as the depth increases under both surroundings. The less $d_{1}$, the higher deviation of peaks, as shown in Fig. 6 b. The absorption peak deviation increases from $2.1 \mathrm{~nm}$ to $2.9 \mathrm{~nm}$ as $d_{1}$ decreases from $360 \mathrm{~nm}$ to $260 \mathrm{~nm}$ within a refractive index deviation 0.02 . The surrounding refractive index sensitivity of the multilayered nanostructure can be improved by optimized and reasonable design of geometry parameters and materials.

\section{CONCLUSIONS}

In summary, a multilayered GWS passive device with notch filtering reflection is proposed by applying a doublelayer grating inside and outside manufactured on silica substrate surface. Characteristics of both resonance peak wavelength situation and FWHM under different structure designing parameters (such as period, thickness, filling factor, et al) are investigated and calculated. Resonance 
filtering bandwidth and attenuation peak can be designed and achieved within any matched wavelength. The absorption spectra possess narrow width and high peak leakage, which exhibits good refractive index sensitivity to surroundings. The wavelength peak shows a red shift as the grating period, thin layer depth, duty cycle or incidence angle increases. The attenuation peaks can be obtained in a wide wavelength range with FWHM of several nanometers to less than one nanometer. The multilayered structure shows a refractive index sensitivity of $220 \mathrm{~nm} / \mathrm{RIU}$ to the surroundings. The larger incident angle makes the larger peak shift, and the longer grating period also makes the larger peak shifts. The simulations and results open a new way in designing, optimizing and fabricating the novel nanostructures integrated photonic devices with such a passive rejection band filtering performance.

\section{Acknowledgements}

This work is supported by the National Scientific Foundation of China (61108024), by the National 863 High-Tech Project of China (2013AA031901) and the Recruitment Program of National 1000 Youth Talents (Qian Sun), by the Natural Science Foundation of Jiangsu Province (BK2011278), by the University Scientific Research Foundation of Jiangsu Province (12KJA480001) and by the project of the Priority Academic Program Development (PAPD) of Jiangsu Higher Education Institutions.

\section{REFERENCES}

1. Wang, S.S., Magnusson, R. Design of Waveguide- Grating Filters with Symmetrical Line Shapes and Low Sidebands Optics Letters 19 1994: pp. 919-921.

2. Tibuleac, S., Magnusson, R. Reflection and Trans- Mission Guided-Mode Resonance Filters Journal of the Optical Society of America A 14 (7) 1997: pp. 1617-1626. http://dx.doi.org/10.1364/JOSAA.14.001617

3. Mizutani, A., Kikuta, H., Iwata, K. Numerical Study on an Asymmetric Guided-Moderesonant Grating with A Kerr Medium for Optical Switching Journal of the Optical Society of America A 22 (2) 2005: pp. 355-360.

4. Niederer, G., Herzig, H.P., $\quad$ Shamir, J., Thiele, H., Schnieper, M., Zschokke, C. Tunable, Oblique Incidence
Resonant Grating Filter for Telecommunications Applied Optics 43 (8) 2004: pp. 1683-1694.

5. Cao, B., Zhang, G., Wang, C., Wang, J., Xu, K. Narrowband Notch Filters with Composite Nanostructure Layers on a GaN-Based Light Emitting Diode Advanced Materials Research 486 2012: pp. 114-118. http://dx.doi.org/10.4028/www.scientific.net/AMR.486.114

6. Lee, Y.C., Huang, C.F., Chang, J.Y., Wu, M.L. Enhanced Light Trapping Based on Guided Mode Resonance Effect for Thin-Film Silicon Solar Cells with Two Filling Factor Grating Optics Express 16 (11) 2008: pp. 7969-7975.

7. Wei, X., Weiss, S.M. Guided Mode Biosensor Based on Grating Coupled Porous Silicon Waveguide Optics Express 19 (12) 2011: pp. $11330-11339$.

8. Hu, M., An, R.D., Zhang, H., Huang, Q.F., Ge, J.H. Experimental Investigation of a Novel Microchip Laser Producing Synchronized Dual-Frequency Laser Pulse with an $85 \mathrm{GHz}$ Interval Laser Physics Letters 10 2013: pp. $015801-015805$.

http://dx.doi.org/10.1088/1612-2011/10/1/015801

9. Moharam, M.G., Gaylord, T.K.T.K. Rigorous CoupledWave Analysis of Metallic Surface-Relief Gratings Journal of the Optical Society of America A 3 1986: pp. $1780-1787$. http://dx.doi.org/10.1364/JOSAA.3.001780

10. Jacob, D.K., Dunn, S.C., Moharam, M.G. Design Considerations for Narrow-Band Dielectric Resonant Grating Reflection Filters of Finite Length Journal of the Optical Society of America A 17 2000: pp. 1241-1249.

11. Doskolovich, L., Kadomina, E., Kadomin, I., Kharitonov, S. Software for Designing and Modeling the Diffraction Gratings Within the Rigorous Electromagnetic Theory Optical Memory and Neural Networks 16 (1) 2007: pp. $24-30$.

12. Zhang, G., Wang, C., Cao, B., Huang, Z., Wang, J., Zhang, B., Xu, K. Polarized GaN-Based LED with an InteGrated Multi-Layer Subwavelength Structure Optics Express 18 (7) 2010: pp. 7019-7030. http://dx.doi.org/10.1364/OE.18.007019

13. Tamulevičius, T., Gražulevičiūtè, I., Urbonas, D., Gabalis, M., $\quad$ Petruškevičius, R., $\quad$ Tamulevičius, $\mathbf{S}$. Numerical and Experimental Analysis of Optical Response of Sub- Wavelength Period Structure in Carbonaceous Film For Refractive Index Sensing Optics Express 22 (22) 2014: pp. $27462-27475$. 